Keio J. Med. 21 : 201-213, 1972

\title{
THYMIC CHANGE IN BEHÇET'S DISEASE
}

\author{
NORIKAZU TAMAOKI*, SONOKO HABU*, HIROSHI YOSHIMATSU**, \\ MASAHARU TSUCHIYA***, HIROSHI WATANABE**** \\ and TADAYOSHI KOBAYASHI*
}

Departments of Pathology; Surgery, and Internal Medicine, School of Medicine,
Keio University, Tokyo and
Department of Pathology, School of Medicine, Fujita Gakuen University, Nagoya

(Received for publication August 14, 1972)

\section{INTRODUCTION}

Behçet's disease is characterized by recurring ocular, oral and genital symptoms ${ }^{1}$ with frequent involvement of the skin, ${ }^{2}$ gastrointestinal tract, ${ }^{3}$ lung, ${ }^{4}$ joints $^{5}$ and nervous system. ${ }^{6}$ Although basic pathological findings have been reported as an uncharacteristic inflammation, ${ }^{3}$ some reports stressed vascular lesions including necrotizing angitis and thrombophlebitis in various organ systems and suggested an allergic nature or relationship to a collagen disease, ${ }^{4,7}$ The viral etiology of Behçet's disease is still inconclusive, but recent reports on the presence of autoantibodies against the oral mucosa indicate that an autoimmune mechanism may be involved in the pathogenesis..$^{9,10}$

Thymic abnormalities such as thymic lymphoid follicles and epithelial change have been reported in systemic lupus erythematosus ${ }^{11}$ and other autoimmune diseases, ${ }^{12,13}$ and it was suggested that the clone reacting against autoantigens was induced by the abnormal immunological function of the thymus. ${ }^{14}$ A review of the literatures has revealed only one report with a histological description of the thymus in Behçet's disease. ${ }^{15}$ The present report concerns the pathological findings on thymuses resected from 22 cases of Behçet's disease.

* Department of Pathology, School of Medicine, Keio University, Tokyo.

** Department of Surgery, School of Medicine, Keio University, Tokyo.

*** Department of Internal Medicine, School of Medicine, Keio University, Tokyo.

**** Department of Pathology, School of Medicine, Fujita Gakuen University, Nagoya. 


\section{MATERIALS AND METHODS}

Cases studied. Thymuses was resected via the suprasternal notch ${ }^{16}$ from twenty-two cases of Behçet's disease. Nineteen patients were treated at the Keio University Hospital and three others were seen at the Neurology Clinic of the National Saitama Hospital by Dr. R. Kodama. Cases were categorized into three groups based upon the route, dose and interval of the given corticosteroids.

A. Cases without history of a systemic administration of steroids. This group includes cases treated solely by the local application of steroids to the conjunctival sac and/or the skin.

B. Cases given steroids temporarily by the systemic route and discontinued at least for three months prior to thymectomy.

C. Cases given steroids systemically at the time of the operation or within three months prior to thymectomy.

Histopathological study. Thymuses were fixed in $10 \%$ formalin and at least two blocks were taken from each lobe. Paraffin suctions were stained with hematoxylin and $\operatorname{cosin}(H \& E), P A S$ and silver impregnation according to the modified Gomori method.

A relative proportion of areas occupied by lymphoid-epithelial tissue and fatty tissue in the thymus was judged microscopically on the sections stained with $\mathrm{H} \& \mathrm{E}$. Degree of involution of the thymus was estimated on the basis of this proportion and expressed in the following four grades. No quantitative measurement was attempted.

Grade 1. Area occupied by lymphoid-epithelial tissue exceeds that occupied by fatty tissue.

Grade 2. Area occupied by lymphoid-epithelial tissue roughly equals that occupied by fatty tissue.

Grade 3 . Area occupied by fatty tissue exceeds that occupied by lymphoidepithelial tissue.

Grade 4 . The thymic cortex is replaced completely by fatty tissue in addition to changes described in grade 3.

Thy thyroid and sternocleidomastoid muscle were biopsied during the operation and their histologies were examined.

Measurement of number and size of thymic lymphoid follicle. The total number of lymphoid follicles found in the sections of each thymus was shown in tables. 
THYMIC CHANGE IN BEHÇET'S DISEASE

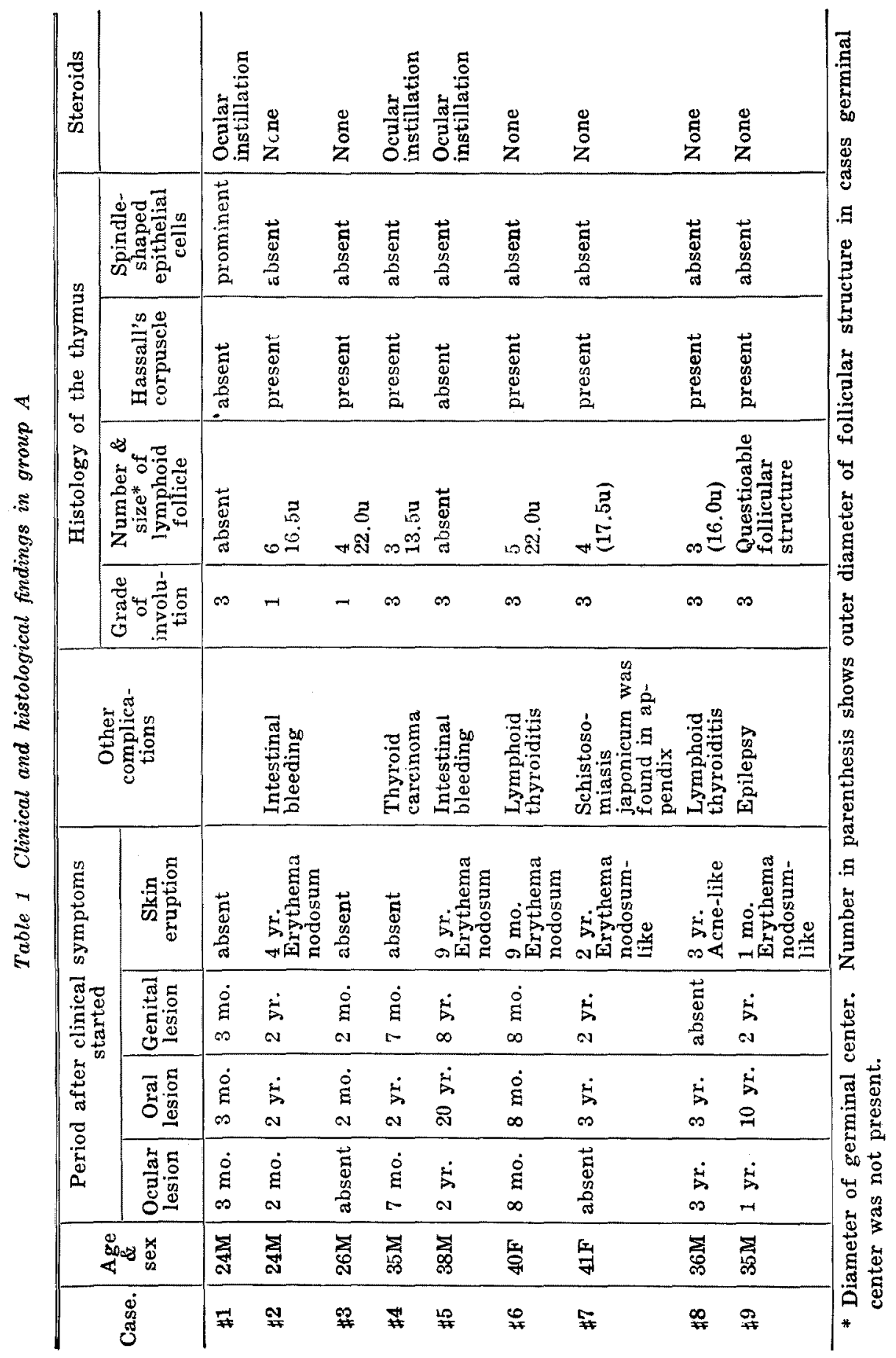




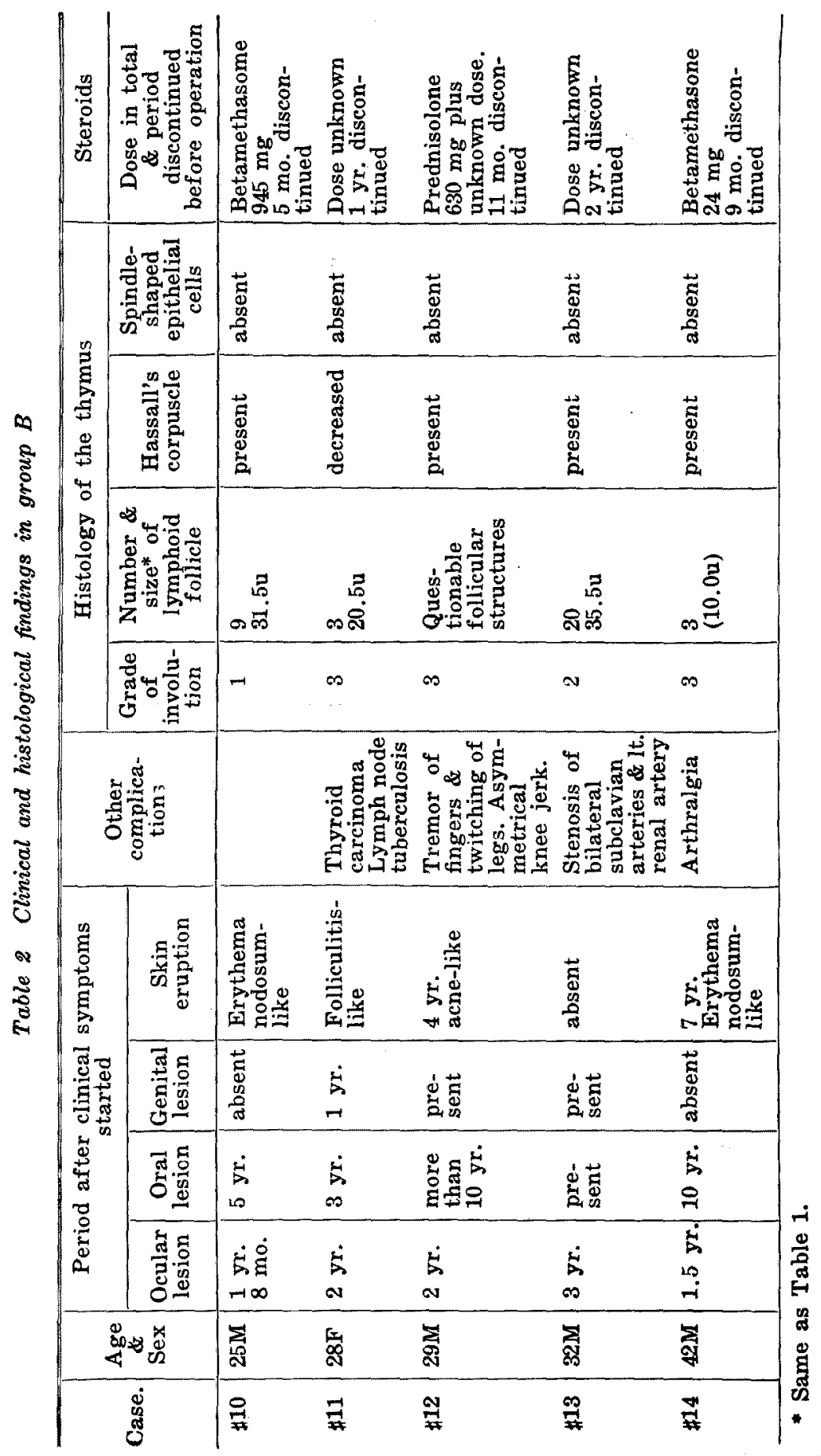




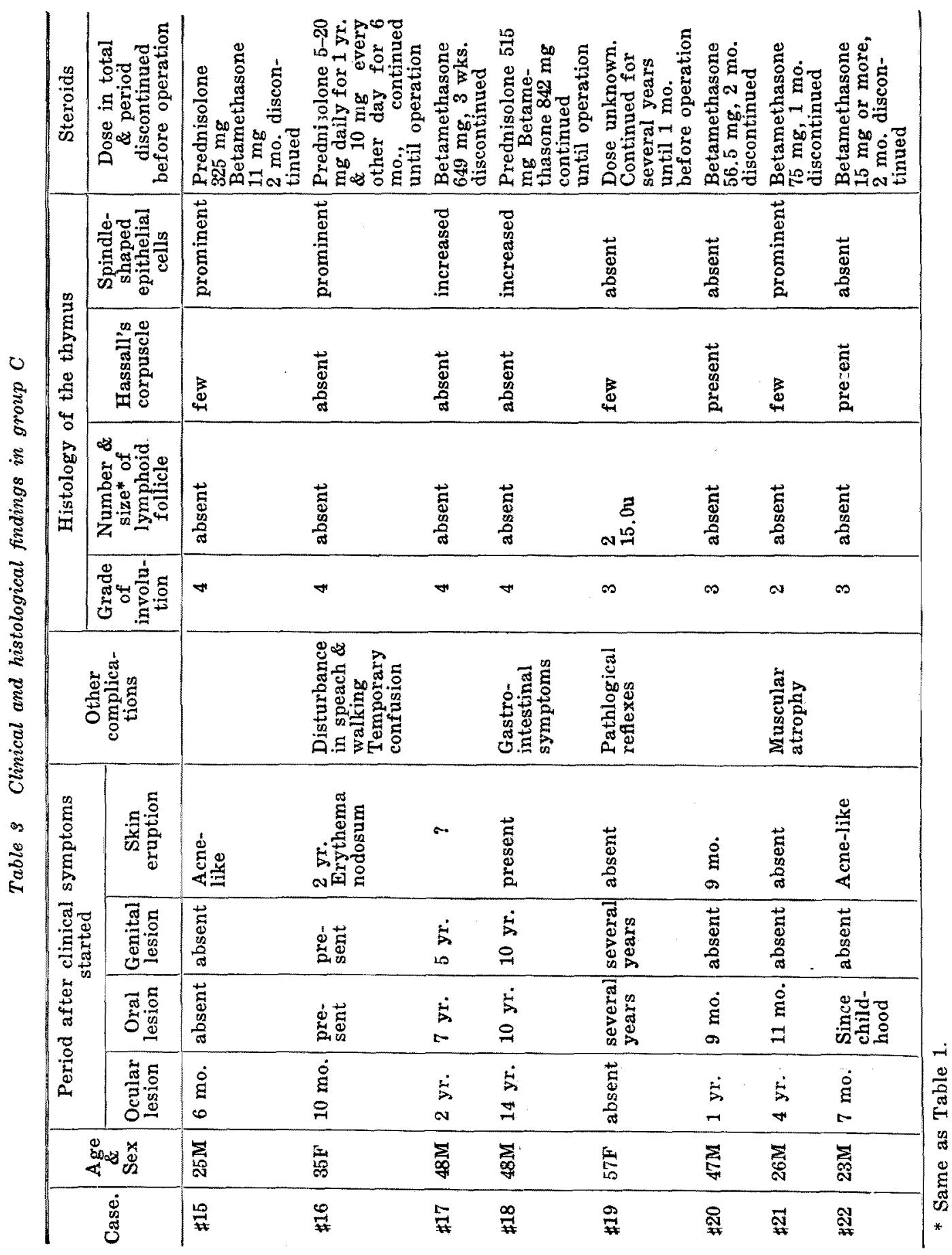


The long and short axes of the largest thymic germinal center in each case were measured microscopically on the sections and their mean values were shown in the table as an indicator of the sizes of the lymphoid follicles. When a germinal center was not present, the outer diameter of the lymphoid follicle was determined. In addition to cases of Behçet's disease, thymuses in the previous paper ${ }^{12}$ were used for comparison.

\section{RESULTS}

The clinical and histological findings were summarized in Table 1 to 3 ,

Involution of the thymus. Degree of involution was varied in group A and $B$, although it seemed to advance with increasing age. Thymuses of the cases given large doses of steroids in group $\mathrm{C}$ showed marked involution with almost a complete disappearance of the cortex. A marked karyorrhexis of cortical lymphocytes was noted in case \#7.

Thymic lymphoid follicle. Thymic lymphoid follicles were found in six cases out of nine in group $A$ and four out of five in group B. Cases \#9 and $\# 2$ showed questionable follicular structures in the medulla. In group $\mathrm{C}$, on the other hand, only two small germinal centers were noted in one case out of eight. Lymphoid follicles were always situated at the junction of the thymic medulla and the interlobular connective tissue similar to those seen in other autoimmune diseases, and most of them contained germinal centers composed of large blastic cells with scattered tingible-body macrophages (Figs. 1 and 2 ). In case $\# 7$ and $\# 14$, lymphoid follicles were composed exclusively of medium-sized lymphocytes and no germinal center was identified.

Table 4

Number and size of thymic lymphoid follicles

\begin{tabular}{l|c|c|c}
\hline & No. cases & $\begin{array}{c}\text { Number of } \\
\text { lymphoid follicle }\end{array}$ & $\begin{array}{c}\text { Size of } \\
\text { lymphoid follicle }\end{array}$ \\
\cline { 2 - 4 } & mean (range) & mean \pm S.D. \\
\hline Behcet's disease & 10 & $5.9(2-20)$ & $20.0 \pm 7.9 \mu$ \\
Myasthenia gravis & 31 & $34.1(2-122)$ & $29.6 \pm 11.5 \mu$ \\
Other autoimmune diseases* & 8 & $24.1(2-115)$ & $29.8 \pm 13.8 \mu$ \\
\hline
\end{tabular}

* SLE 2, Autoimmune hemolytic anemia 2, Hashimoto throiditis 3, Colitis ulcerosa 1. 
The number of lymphoid follicles ranged from 2 to 20 (mean 5.9) in Behçet's disease, 1 to 122 (mean 34.1) in myasthenia gravis and 2 to 115 (mean 24.1) in other autoimmune diseases. Although comparison with other diseases was difficult because the number of blocks taken from resected thymuses was not standarized, the number of lymphoid follicles seemed to be considerably lower in Behçet's disease than in other diseases related to autoimmunity (Table 4). The size of lymphoid follicle was also small in Behçet's disease as compared with myasthenia gravis $(\mathrm{P}<0.02)$ and other autoimmune diseases (statistically not significant) (Table 4).

Thymic epithelial cells. Hassall's corpuscles were numerous in younger cases under fourty years old in group A and B. In the older cases, focal collections of epithelial cells which did not form Hassall's corpuscle were frequently observed in the medulla of some lobules. These cells were polygonal, cylindrical or elongated in shape and possessed relatively abundant cytoplasm, but did not show a tendency of keratinization (Fig. 3).

In five cases out of eight in group $\mathrm{C}$, on the other hand, Hassall's corpuscle disappeared and spindle-shaped epithelial cells were prominent in the medulla (Fig. 4). These cells had scanty cytoplasm and resembled those observed in the thymuses of SLE and thymoma of the spindle-cell type. Furthermore, in the thymus of case \#1, which had not been treated with a systemic administration of steroids, Hassall's corpuscle was not present and spindle-shaped epithelial cells predominated in the medulla (Fig. 6). A few remnants of Hassall's corpuscles consisting of keratinized epithelial cells were circumscribed by foreign-body giant cells (Fig. 5).

No vascular lesion or acute inflammatory change was noted despite careful examination. The intercellular and perivascular hyalinization observed in the thymuses of SLE and myasthenia gravis were not found in the present series.

Findings on the thyroid. A firm nodule about $1 \mathrm{~cm}$ across was found in the right lobe of the thyroid of case \#4 at the time of operation and an examination of frozen sections revealed a papillary carcinoma. No intrathyroidal spread or distant metastasis was found in the resected right lobe and cervical lymph nodes. In addition, in case \#11, papillary and follicular carcinoma measuring about $2 \mathrm{~cm}$ in diameter was found in the left upper pole of the thyroid with metastasis to the cervical and upper mediastinal lymph nodes. The latter case had a history of irradiation to left cervical region due to tuberculous lymphadenitis during her childhood. Biopsy of the axillary lymph nodes, one year prior to thymic resection, revealed active tuberculosis.

There was no remarkable change in the thyroid of the other cases except for 
cases \#6 and \#8 which showed moderate lymphoid thyroiditis.

\section{DISCUSSION}

A high incidence of thymic lymphoid follicle was noted in patients with Behçet's disease who did not receive steroids systemically $(6 / 9,66 \%)$, and in those where steroids had been discontinued for more than three months before resection $(4 / 5,80 \%)$, although the number of follicles was small. The incidence of thymic lymphoid follicle has been reported in $83.8 \%$ of the patients with myasthenia gravis, and in $39.1 \%$ of the patients with other autoimmune diseases, whereas the occurrence in diseases not related to autoimmunity was rare.12 These findings suggest that the thymus may be implicated in the pathogenesis of Behçet's disease as well as in other autoimmune diseases. Immunological surveys revealed that serum autoantibody and the lymphocytes sensitized against autoantigens may be concerned with the development of oral ulcerations and intestinal lesions in Behçet's disease. ${ }^{9,17,18,19}$ Significance of the thymic lymphoid follicle in autoimmune disease is, at present, unknown. Although some authors related it to abnormal, self-reacting clones, ${ }^{14}$ occurrence of the thymic lymphoid follicle in mice of the leukemic strain, $\mathrm{AKR}^{20}$ as well as in the autoimmune strain, NZB seems to indicate that a virus may have been induced to develop the lymphoid follicle in the thymus. Both of the two alternatives are probable etiological factors in Behçet's disease. A relative small number of lymphoid follicles and the absence of hyalinization in the thymus might indicate that the thymic changes in Behçet's disease is not as intense as in SLE and myasthenia gravis.

A low incidence of thymic lymphoid follicle was noted in patients receiving steroids at or within three months prior to the resection. Futagami et al15 failed to confirm any abnormality in the thymus weighing $46 \mathrm{~g}$, removed from a 9 year-old boy with Behçet's disease. This patient had also received $840 \mathrm{mg}$ of prednisolone and $24 \mathrm{mg}$ of betamethasone before thymectomy. Hutchins and Harvey ${ }^{21}$ reported the absence of germinal center in the autopsied cases of SLE received an intensive steroid therapy. A study of the experimental thymic lymphoid follicle shows that the development of lymphoid follicle requires an interaction of lymphocytes both in the thymic medulla and in the peripheral circulation, providing the antigen is present in the thymus. ${ }^{22}$ It is, therefore conceivable that lympholytic corticosteroids prevented the development of lymphoid follicle by decreasing the lymphocyte population in the thymus and in the circulation.

It is interesting that thymic lymphoid follicles were found more frequently in patients who discontinued steroids over 3 months than in those of the non- 
steroid group. It is, therefore, indicated that steroids supress the occurrence of lymphoid follicles in the thymus, but cannot prevent their recurrence after withdrawal.

The focal collection of epithelial cells not comprising Hassall's corpuscle does not seem to be specific for the basic disease, because such cells are frequently seen in the involuted thymus biopsied from various conditions.

Hyperplasia of spindle-shaped epithelial cells, as described in SLE by Hutchins and Harvey, was an unusual finding in the biopsied thymus. In a survey of more than 180 biopsied and resected thymuses, spindle-shaped epithelial cells were prominent in patients with SLE, hypothyroidism, uremia due to severe pyelonephritis and colitis ulcerosa treated with steroids other than Behçet's disease (unpublished data). The cause of this change in Behçet's disease is not clear. No case in the present series was suffering from hypothyroidism. A large dose of steroids may have affected the thymic epithelium to change into a spindle-cell type. However, an examination of resected thymuses from the patients who were transplanted renal allografts and treated with large doses of steroids over one year did not reveal hyperplasia of spindle-shaped epithelial cells. Alternatively, this change might be related to the basic condition in Behçet's disease, because hyperplasia of spindle-shaped epithelial cells were found also in a case not treated with steroids. This case $(\# 1)$ was thymectomized in the period of exacerbation of eye symptoms started three months ago, and the lesion was progressive. Furthermore, a degenerative change of the thymic epithelium has been reported as an initial lesion preceding the appearance of autoantibodies in NZB, NZW and their $F_{1}$ hybrid mice. ${ }^{23}$ It is likely, therefore, that thymic epithelial cells in Behçet's disease and SLE are liable to degenerate and to transform into a spindle-cell type, and that steroids and other unrecognized factors might accelerate this change.

The association of thyroid carcinoma in Behçet's disease has not been reported. Papastestas et al ${ }^{24}$ reported an unusually high occurrence of malignant growth among patients with myasthenia gravis in which thymic lymphoid follicles are known to be very frequent. It is suggested that the thymic changes in Behçet's disease might play some role in the altered immunological surveillance and oncogenesis. A further study is required to clarify whether their co-existence is incidental or not.

The lymphoid thyroiditis has also been frequently found in association with myasthenia gravis and may be a manifestation of the autoimmune reaction against multiple organs. 


\section{SUMMARY}

Histologies of the thymuses resected from 22 cases of Behçet's disease were described. The incidence of thymic lymphoid follicle was $66 \%(6 / 9)$ in patients not treated with systemic steroid therapy, $80 \%(4 / 5)$ in those who discontinued steroids for more than three months before thymectomy, and $13 \%(1 / 8)$ in one who was treated at or within three months before the resection. Hyperplasia of spindle-shaped epithelial cells with the disappearancè of Hassall's corpuscle was noted in five out of eight cases treated and in one case not treated with systemic steroid administration. It was suggested that the presence of thymic lymphoid follicles and spindle-shaped epithelial cells might be related to basic abnormalities in the pathogenesis of Behçet's disease. Two cases associated with thyroid carcinoma and two cases with lymphoid thyroiditis were also reported.

\section{REFERENCES}

1. Behçet, H. (1937) Úber rezidivierende, aphtöse, durch ein Virus verursachte Geschwüre am Mund, am Auge und an den Genitalien. Derm. Wsch. 105: 11521157.

2. Nishiyama, S. (1959) Klinische Beobachtungen über Behçet'scher Krankheit. Jap. J. Dermatol. 69: 1139-1185. (in Japanese)

3. B $\phi$ e, J., Dalgaard, J. B., and Scott, D. (1958) Mucocutaneus-ocular syndrome with intestinal involvement. Am. J. Med. 25: 857-867.

4. Sulheim, O., Dalgaard, J. B., and Andersen, S. Ry. (1958) Behçet's syndrome. Report of case with complete autopsy performed. Acta path. microbiol. Scand. 45: $145-158$.

5. Strachan, R. W. and Wigzell, F, W. (1963) Polyarthritis in Behçet's multiple symptom complex. Ann. Rheum. Dis. 22: 26-35.

6. Wolf, S. M., Schotland, D. L., and Phillips, L. L. (1965) Involvement of nervous system in Behçet's syndrome. Arch. Neurol. 12: 315-325.

7. Shikano, S. (1959) Pathology of the eye with Behçet's syndrome (abstract in English). Jap. clin. Ophtholm. 13: 119-134.

8. O'Duffy, J. D., Caney, J. A., and Deodhar, S. (1971) Behçet's disease, Report of 10 cases, 3 with new manifestations. Ann. int. Med. 75: 561-570.

9. Oshima, Y., Shimizu, T., Yokohari, R., Matsumoto, T., Kano, K., Kagami, T., and Nagaya, H. (1963) Clinical studies on Behçet's syndrome. Ann. Rheum. Dis. 22: $36-45$.

10. Lehmer, T. (1967) Behçet's syndrome and autoimmunity. Brit. Med. J. 1: 465-467.

11. Mackay, I. R. and de Gail, P. (1963) Thymic "germinal center" and plasma cells in systemic lupus erythematosus. Lancet 2: 66-67.

12. Habu, S., Kameya, T., and Tamaoki, N. (1971) Thymic lymphoid follicles in autoimmune diseases. I. Quantitative studies with special reference to myasthenia gravis. Keio J. Med. 20 : 45-56.

13. Okabe, H. (1967) Thymic lymphoid follicles: A histological study of 1356 autopsy cases. Acta path. Jap. 16: 109-130. 
14. Burnet, F. M. (1962) Role of the thymus and related organs in immunity. Brit. Med. J. 2: 807-811.

15. Futagami, T., Saito, K., Saito, M., Saneto, M., and Fujioka, K. (1969) New therapeutic attempts in Behçet's diseases (Abstract in English). Jap. J. Clin. Ophthalm. 23: 557-564.

16. Yoshimatsu, H., Tsuchiya, M., Kamegaya, K., and Oda, M. (1971) Mediastinoscopy and thymictomy via the suprasternal notch. Mediastinoscopy. Proceedings of an International Symposium Odense University, June, 1970. Eds. Jepsen, O,, \& Sorensen, H. R. pp. 94-96. Odense University Press.

17. Lehmer, T. (1969) Characterization of mucosal antibodies in recurrent aphthous ulceration and Behçet's syndrome. Arch. oral Biol. 14: 843-853.

18. Lehmer, T. (1969) Pathology of recurrent oral ulceration in Behçet's syndrome: Light Electron and Fluorescence microscopy. J. Pathol. 97: 481-494.

19. Saito, T., Honma, T., Sato, T., and Fujioka, Y. (1971) Autoimmune mechanisms as a probable aetiology of Behçet's syndrome, an electron microscopic study of the oral mucosa. Virchow's Arch. (Path. Anat.) 353: 261-272.

20. Metcalf, D. (1966) Histologic and transplantation studies on preleukemic thymus of the AKR mouse. J. Nat. Cancer Inst. 37: 425-442.

21. Hatchins, G. M. and Harvey, A. M. (1964) Thymus in systemic lupus erythematosus. Bull. Johns Hopkins Hosp. 115: 355-378.

22. Habu, S. (1972) Experimental production of lymphoid follicles in thymus by horseradish peroxidase. Acta Path. Jap. 22: 681-695.

23. de Vries, M. J. and Hijmans, W. (1967) Pathological changes of thymic epithelial cells and autoimmune diseases in $\mathrm{NZB}, \mathrm{NZW}$ and $(\mathrm{NZB} \times \mathrm{NZW}) \mathrm{F}_{1}$ mice. Immunology 12: 179-196.

24. Papastestas, A. E., Osserman, K. E., and Kark, A. E. (1972) The relationship between thymus and oncogenesis. A study of the incidence of non-thymic malignancy in myasthenia gravis. Brit. J. Cancer 25: 635-645. 


\section{EXPLANATION OF PLATES}

Fig. 1. Case \#10. 25 y.o. Male. The thymus resected one year after the ocular symptom started shows two thymic lymphoid follicles with germinal center in the medulla (arrows). Steroid therapy had been discontinued for 5 months before thymectomy. H. \& E. $\times 76$.

Fig. 2. Case \#2. 24 y.o. Male. Thymic germinal center and crystic Hassall's corpuscle found in the resected thymus 2 months after the ocular symptim started. No steroid had been administered. H. \& E. $\times 170$.

Fig. 3. Case \#4. 35 y.o. Male. Focal collection of non-keratinizing epithelial cells with relatively abundant cytoplasm were seen in the medulla. The thymus was resected 7 months after the ocular symptom started and complication of thyroid carcinoma was found. No steroid therapy was attempted. H. \& E. $\times 346$.

Fig. 4. Case \#15. 25 y.o. Male. Complete involution of the cortex, absence of Hassall's corpuscle and prominent spindle-shaped epithelial cells in the thymus resected 6 months after the ocular symptom started. Intensive steroid therapy had been continued until operation. H. \& E. $\times 346$.

Fig. 5. Case \#1. 24 y.o. Male. Marked involution of the cortex and foreign-body reaction around the remnants of Hassall's corpuscle in the thymus resected 3 months after the onset of ocular symptom which was progressive. No systemic steroid therapy was done. H. \& E. $\times 76$.

Fig. 6. Case \#1. A higher magnification shows prominent spindle-shaped epithelial cells and disappearance of Hassall's corpuscle. H. \& E. $\times 346$. 


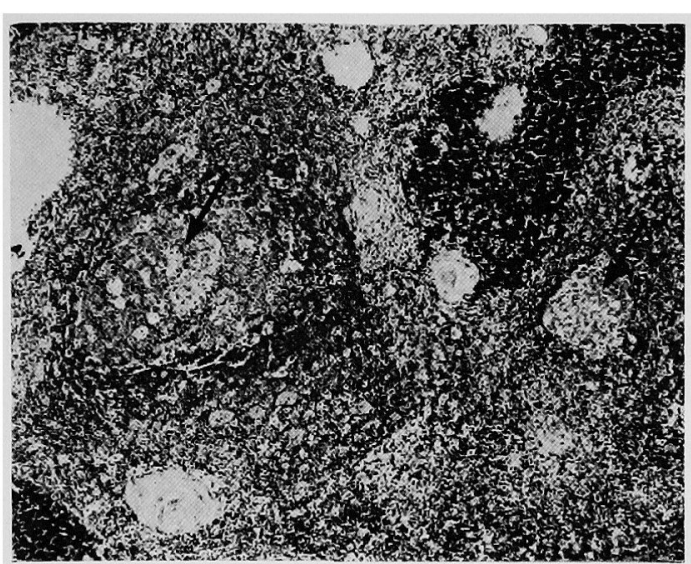

Fig. 1

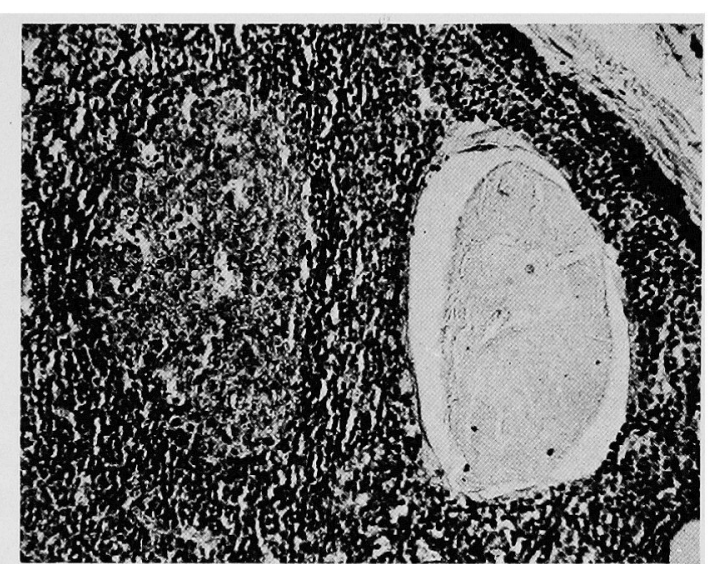

Fig. 2

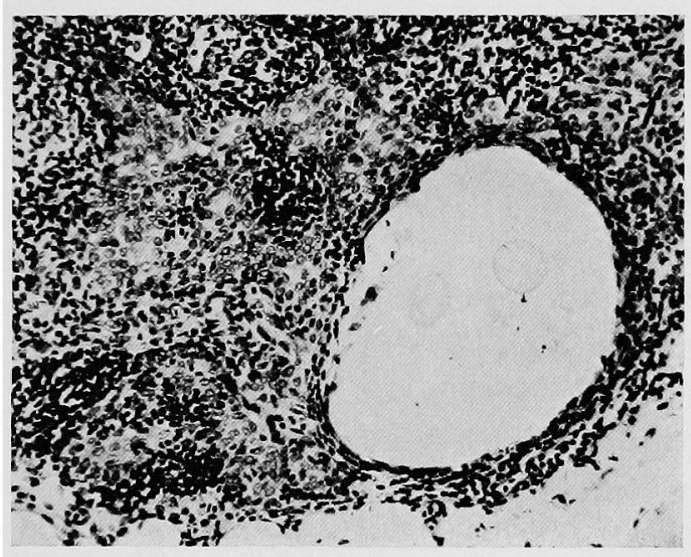

Fig. 3

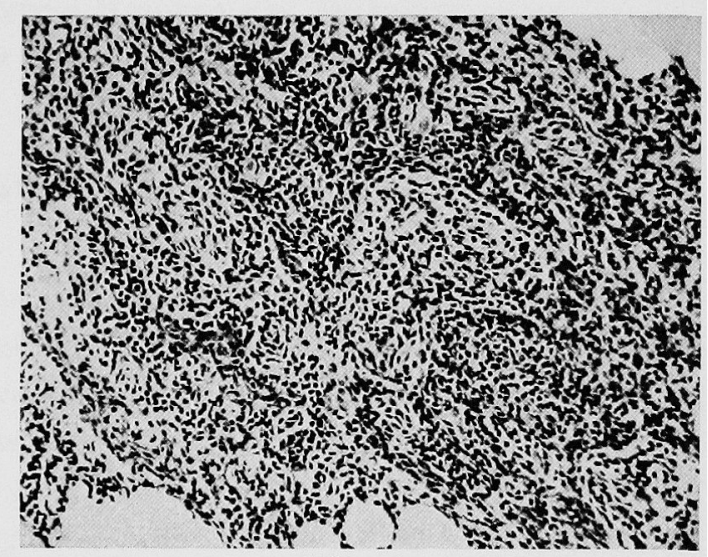

Fig. 4

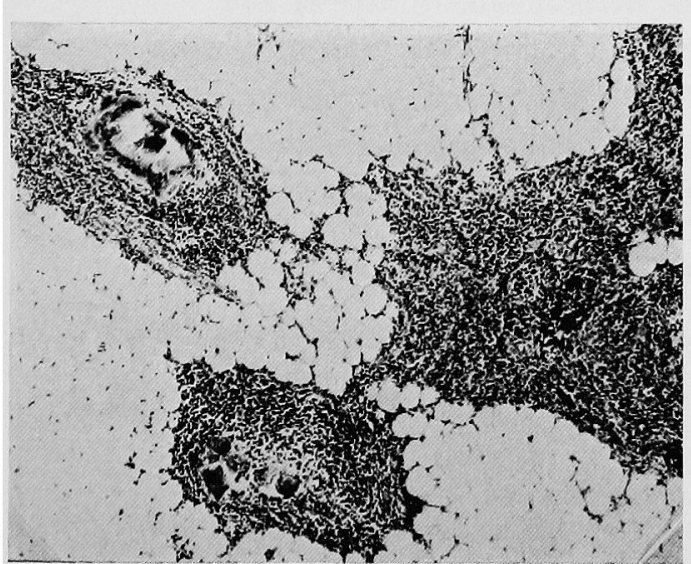

Fig. 5

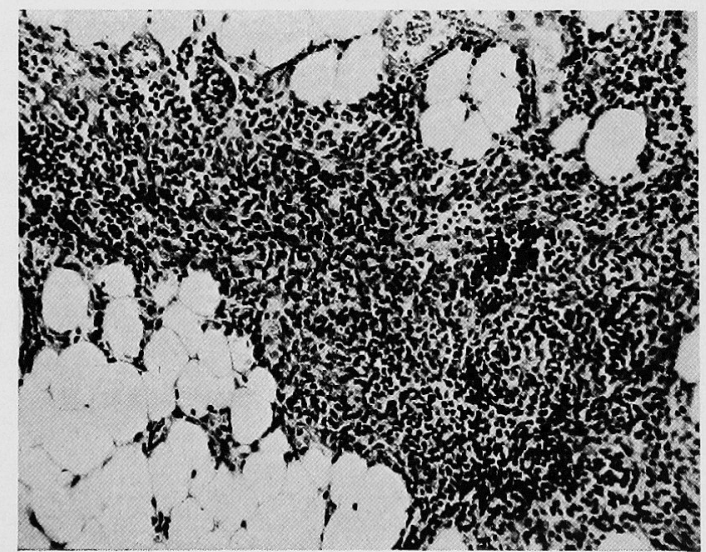

Fig. 6 